ISSN 0819-2642

ISBN 9780734040152

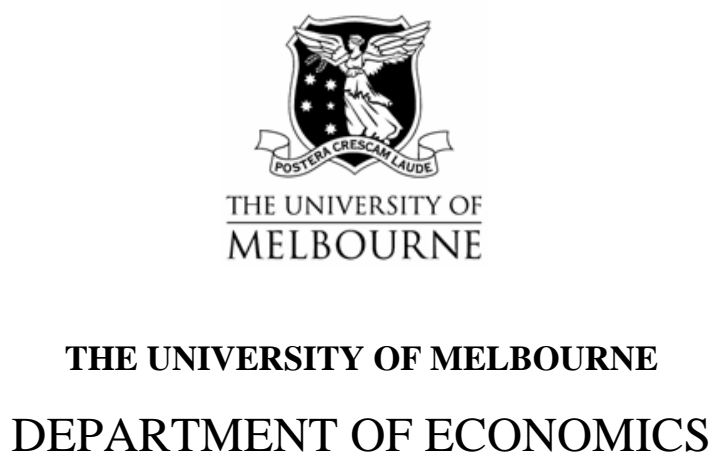

RESEARCH PAPER NUMBER 1049

August 2008

\title{
Directed Search, Unemployment and Public Policy
}

by

Benoit Julien, John Kennes, Ian King \& Sephorah Mangin

Department of Economics

The University of Melbourne

Melbourne Victoria 3010

Australia. 


\title{
Directed Search, Unemployment and Public Policy
}

\author{
Benoît Julien John Kennes Ian King \\ Sephorah Mangin
}

August 2008

\begin{abstract}
We examine the effects of public policy parameters in a simple directed search model of the labour market, and contrast them with those in standard random matching models with Nash bargaining. Both finite and limit versions of the directed search model are considered, and the value of the limit model as an approximation of the finite one is assessed. As with the random matching model, job creation is the key channel through with the policy parameters effect the equilibrium of the directed search model. Both comparative static effects of the policy parameters and optimal configurations are identified.
\end{abstract}

JEL Codes: E24, J31, J41, J64, H20, D44

Benoît Julien: Department of Economics, University of New South Wales

John Kennes: Department of Economics, Aarhus University

Ian King: Department of Economics, University of Melbourne

Sephorah Mangin: Department of Economics, University of Melbourne 


\section{INTRODUCTION}

Since Pissarides' (1985) classic paper, it has been well known that the existence of search frictions can have a significant influence on both the positive and normative effects of tax and subsidy structures in labour markets. Search frictions convey a measure of local market power that allows for non-competitive pricing even in large markets. This, in principle, allows for the existence of profits for firms. However, with entry, these profits are driven to zero in equilibrium - a condition that determines the number of participants in a market. As a consequence, entry is a key margin in search models. In the context of the labour market, different tax and subsidy structures affect this margin and, thereby, job creation, unemployment, and other endogenous variables, in a way quite different to competitive models.

Traditionally, search models of the labour market have modelled the matching process, between workers and vacancies, using the matching function approach. In this approach, people meet bilaterally, where the probability of a meeting is determined according to a matching technology which has unemployed workers and unfilled vacancies as inputs, and matches as outputs. By assumption, this technology is increasing in both arguments, concave, and, usually, is presumed to have constant returns to scale. In these models, the division of the surplus from a match (i.e., wage determination) is usually modelled by appealing to the Nash bargaining solution. ${ }^{1}$

A well-known feature of these models is that their equilibria are not, in general, constrained-efficient. In particular, the equilibrium allocation does not maximize expected surplus, even conditional on matching frictions, except under a very special condition, known as the "Hosios condition", where the elasticity of the matching function with respect to unemployed workers happened to equal the value of workers' bargaining power in the generalized Nash bargaining solution. ${ }^{2}$ Since both the matching elasticity and bargaining power are independent parameters in the model, there is nothing in the

\footnotetext{
${ }^{1}$ All of this is made explicit and clear in Pissarides (1985). However, see Rogerson, Shimer and Wright (2005) for an excellent survey and discussion of these models.

${ }^{2}$ See Hosios (1990).
} 
model to ensure that this condition will be met. More generally, this inefficiency is due to a hold-up problem in these models: firms must pay a cost, up-front, to create a vacancy, in order to enter the market. Upon meeting a worker, this cost is sunk from the point of view of the firm - a fact that the worker can exploit. Firms' incentives to enter, therefore, are diminished. This effect is offset only when the Hosios condition applies.

Although this generic inefficiency was not the focus of Pissarides' (1985) original study, it has, quite naturally, become central in subsequent studies. Boone and Bovenberg (2002), for example, examine distortionary tax and subsidy structures that can, among other things, restore efficiency in this environment. Hungerbühler et al (2006), when considering redistributive taxes in the face of heterogeneous productivities with search, resort to imposing the Hosios condition, in order to abstract away from these issues and concentrate on the redistributive trade-offs stressed by Mirrlees (1971). Lehmann and van der Linden (2007) argue that, due to the hold-up problem, in the absence of informational asymmetries, the optimal marginal wage tax is $100 \%$.

In recent years, a new class of search-theoretic models of the labour market has appeared in which agents in the model are able to observe the location and characteristics of other agents, and choose who to approach, rather than simply accept arbitrary assignment by a matching technology. Due to the fact that agents in these models are directed by the characteristics they observe, this class of models has come to be known as "directed search" theory. At the heart of most of these models is a simple coordination problem, which can lead to multilateral meetings: agents on one side of the market can be matched with more than one agent from the other side. This feature opens the door to alternative wage determination mechanisms (i.e., other than bilateral bargaining). For example, Montgomery (1991) and Burdett, Shi and Wright (2001) consider wage-posting, where each vacancy posts a wage, which they commit to, no matter how many workers approach them. Alternatively, in Julien, Kennes, and King (2000a), workers auction off their labour services, with the wage depending on how many firms approach them. ${ }^{3}$

\footnotetext{
${ }^{3}$ For a survey of directed search, see King (2003) or Shi (2008).
} 
In general, in both of these pricing environments, with directed search, the hold-up problem still exists. However, this problem disappears in the limit when the size of the market approaches infinity - regardless of which of the two wage determination mechanisms (wage posting or auctions) are used. ${ }^{4}$ Thus, in markets of infinite size, equilibria in directed search models of this type are constrained-efficient. This property makes these models qualitatively different from the random matching models (mentioned above) where markets are always assumed to be infinitely large, but constrainedefficiency is obtained if and only if the Hosios condition is imposed.

In this paper we examine the effects of a tax and subsidy scheme, similar to the scheme considered in Pissarides (1985) original study, but in a directed search environment, based on Julien, Kennes, and King (2000a). Bearing in mind the efficiency issue, we consider two variants of the model: with finite and infinite numbers of agents. In the absence of any tax parameters, the equilibrium of the infinitely large economy (which we will refer to as the "limit economy") is constrained efficient, but the equilibrium of the finite-sized economy (which we will refer to as the "finite economy") is not. Clearly, all economies in the real world are finite. The usefulness of the limit economy, we believe, is as an approximation of the finite one. (As will be seen below, the limit economy is significantly easier to work with than the finite one.) Therefore, we also use numerical simulations to ascertain the accuracy of this approximation, as a function of the size of the economy.

Conceptually, two different types of policy questions are considered here. The first is positive in nature: what are the comparative static effects, associated with changes in the relevant policy parameters, on the equilibrium values of the variables? (This is, essentially, the question that Pissarides (1985) considered in the context of random matching.) The second is normative: given that equilibrium allocations in these models involve ex post income inequalities, can we find a configuration of the policy parameters

\footnotetext{
${ }^{4}$ See Julien, Kennes, and King (2000b) for details. See, also, Albrecht, Gautier, and Vroman (2006), which provides a synthesis of these two pricing approaches.
} 
that eliminates this inequality, without disturbing the efficiency properties of the equilibrium?

On the whole, the comparative static effects here are similar to those found in Pissarides (1985), both for the finite and limit economies (although the precise causal mechanisms are different). The normative results also bear a resemblance to those found in Lehmann and van der Linden (2007): the case for a 100\% marginal tax rate is made, (above a certain income level). However, the rationale for this result, in this study, reflects a concern for both efficiency and equity, rather than efficiency alone (as in theirs). In particular, in the limit economy here, as mentioned above, the equilibrium is efficient in absence of any taxes (unlike in the Lehmann and van der Linden (2007) model). In this model, the only role for taxes is to ameliorate ex post inequalities, and the $100 \%$ tax rate, in combination with appropriate configurations of the other policy parameters, achieves this while preserving ex ante efficiency.

The remainder of the paper is organized as follows. Section 1 presents, and characterizes the equilibrium of, the finite economy. Section 2 then presents analogous results for the limit economy. Section 3 contains the comparative static results for both economies. In Section 4, we consider social optima in both economies and, in Section 5, we report the results from the numerical approximation exercise. The conclusions from the study are summarized in Section 6, and the Appendix contains proofs of some of the propositions in the paper. 


\section{THE EXACT (FINITE) ECONOMY}

We consider a simple static economy with a fixed number $N \in \mathbb{N}$ of identical, risk neutral, job candidates where each candidate has one indivisible unit of labor to sell. ${ }^{5}$ There are $M \in \mathbb{N}$ vacancies, where $M$ is determined by free entry. The output from a worker is $y_{0}=0$ if unemployed and $y_{1}=y>0$ if employed. It costs an amount $k$ to create a vacancy, where $k \in[0, y]$. As is standard in this static model, each vacancy can approach, at most, one candidate.

The government in this economy controls four variables: the benefits paid to workers who are unemployed at the end of the period $(\theta \in[0, y])$, an employment subsidy paid to firms that hire workers $(\sigma \in[0, y])$, an income tax rate $(\tau \in[0,1])$, and a level of nontaxable income $(\omega \in[0, y])$.

The order of play is as follows. First, the government sets the values of its policy parameters $(\theta, \sigma, \tau, \omega)$. Then, given $N$ job candidates, $M$ vacancies enter the market. Once the number of entrants has been established, candidates choose their reserve wages $r_{n}$ where $n \in\{1,2, \ldots, N\}$ is used to index candidates. Observing the reserve wages, vacancies then choose which candidate to approach. Once vacancies have been assigned to candidates, wages are determined through the bidding game: candidates sell their labour services to the highest bidder. We solve the model using backwards induction.

\subsection{The Bidding Game}

Here, we take as given the policy parameters $(\theta, \sigma, \tau, \omega)$, the number of vacancies $M$, the vector of reserve wages $\mathbf{r}$, and the assignment of vacancies to candidates. Let $m_{n} \in\{1,2, \ldots, M\}$ denote the number of vacancies bidding for candidate $n$. Let $w\left(r_{n}, m_{n}\right)$

\footnotetext{
${ }^{5}$ In this paper, as in Boone and Bovenberg (2002) and Hungerbühler et al (2006), we abstract away from dynamic issues. This allows for the clearest possible exposition of the effects that we focus on here - in both the small and large economies. For a dynamic version of the large economy, but without any public policy instruments, see Julien, Kennes, and King (2005b).
} 
denote the before-tax equilibrium wage obtained by candidate $n$. The before-tax outside option for each candidate is the benefit payment $\theta$, and the outside option for the vacancy is zero. Without loss of generality, we assume that $r_{n} \in[\theta, y+\sigma]$ for all $n=1,2, \ldots, N$.

For any given $r_{n} \in \mathbf{r}$, the ascending-bid auction generates the following before-tax wage distribution:

$$
w\left(r_{n}, m_{n}\right)=\left\{\begin{array}{ccc}
0 & \text { if } & m_{n}=0 \\
r_{n} & \text { if } & m_{n}=1 \\
y+\sigma & \text { if } & m_{n}>1
\end{array}\right.
$$

Clearly, the wage for any candidate who is unemployed at the end of the period will be zero. If more than one vacancy approaches a candidate, then Bertrand competition drives the wage up to the point where the candidate receives all the output from the production, $y$, plus all of the subsidy, $\sigma$, that vacancies receive when they hire workers. Otherwise, if exactly one vacancy approaches a candidate, the wage paid is simply the reserve wage $r_{n}$.

Let $b\left(r_{n}, m_{n}\right)$ denote the payoff obtained by candidate $n$ after both taxes and government benefits. The above wage distribution $w\left(r_{n}, m_{n}\right)$ generates the following after-tax payoffs:

$$
b\left(r_{n}, m_{n}\right)=\left\{\begin{array}{clc}
\min \{\theta(1-\tau)+\tau \omega, \theta\} & \text { if } & m_{n}=0 \\
\min \left\{r_{n}(1-\tau)+\tau \omega, r_{n}\right\} & \text { if } & m_{n}=1 \\
(y+\sigma)(1-\tau)+\tau \omega & \text { if } & m_{n}>1
\end{array}\right.
$$

An unemployed candidate's after-tax payoff will be $\theta(1-\tau)+\tau \omega$ provided that the level of non-taxable income $\omega$ is no greater than the benefit payment $\theta$. If, however, $\omega>\theta$ (or unemployment benefits are not taxable), then unemployed candidates receive $\theta$ after tax. Thus, the after-tax payoff for unemployed workers is $\min \{\theta(1-\tau)+\tau \omega, \theta\}$. 
A candidate who is approached by exactly one vacancy will receive an after-tax payoff of $r_{n}(1-\tau)+\tau \omega$ provided that $\omega \leq r_{n}$; otherwise, the payoff is simply $r_{n}$. In general, then, the candidate's after-tax payoff is $\min \left\{r_{n}(1-\tau)+\tau \omega, r_{n}\right\}$.

Since $\omega \leq y$ by assumption, the after-tax payoff to a candidate who is approached by more than one vacancy is simply $(y+\sigma)(1-\tau)+\tau \omega$.

\subsection{Vacancies' Choice of Candidate to Approach}

Given the policy parameters $(\theta, \sigma, \tau, \omega)$, the number of vacancies $M$, and the vector of reserve wages $\mathbf{r}$, each vacancy chooses which candidate to approach. For any $r_{n} \in \mathbf{r}$ let $\mathbf{r}_{-n}$ denote the vector of wages $\left(r_{1}, r_{2}, \ldots, r_{n-1}, r_{n+1}, \ldots, r_{N}\right)$. Let $p_{n}\left(r_{n}, \mathbf{r}_{-n}\right)$ denote the probability that any particular vacancy approaches candidate $n .^{6}$ Thus, for each vacancy:

$$
\sum_{n=1}^{N} p_{n}\left(r_{n}, \mathbf{r}_{-n}\right)=1
$$

Also, given $m_{n}$ identical offers, with symmetry, the probability that candidate $n$ accepts any particular offer is $1 / m_{n}$. By equation (1.1) above, if the vacancy is alone when it approaches candidate $n$, it must pay $r_{n}$, so its payoff will be $y+\sigma-r_{n}$. Alternatively, if the vacancy is not alone when approaching candidate $n$, then it must pay $y+\sigma$, so its payoff will be zero. Moreover, in any symmetric equilibrium, the probability that a vacancy will be alone when it approaches candidate $n$ is $\left(1-p_{n}\left(r_{n}, \mathbf{r}_{-n}\right)\right)^{M-1}$. Thus, before knowing $m_{n}$, a vacancy's expected payoff from approaching candidate $n$ is:

$$
\Pi_{n}\left(r_{n}, \mathbf{r}_{-n}\right)=\left(1-p_{n}\left(r_{n}, \mathbf{r}_{-n}\right)\right)^{M-1}\left(y+\sigma-r_{n}\right)
$$

\footnotetext{
${ }^{6}$ Since all vacancies are homogeneous and we focus on symmetric equilibria, to save on notation, we do not index their strategies.
} 
In a mixed strategy equilibrium, each vacancy chooses $p_{n}\left(r_{n}, \mathbf{r}_{-n}\right), n=1,2, \ldots, N$, so that $\Pi_{n}=\Pi, \forall n$. Let $p_{-n}\left(r_{n}, \mathbf{r}_{-n}\right)$ denote the symmetric mixed strategy probability assigned to all other candidates, then equation (1.2) becomes:

$$
p_{-n}\left(r_{n}, \mathbf{r}_{-n}\right)=\frac{1-p_{n}\left(r_{n}, \mathbf{r}_{-n}\right)}{N-1}
$$

Using this, together with equation (1.3), and the condition $\Pi_{n}=\Pi$, assuming that the reserve wage for all candidates other than $n$ is equal to some value $r$ (so $\left.p_{n}\left(r_{n}, \mathbf{r}_{-n}\right)=p_{n}\left(r_{n}, r\right)\right)$ we obtain:

$$
p_{n}\left(r_{n}, r\right)=1-\frac{N-1}{1+(N-1)\left(\frac{y+\sigma-r_{n}}{y+\sigma-r}\right)^{\frac{1}{M-1}}}
$$

Equation (1.4) presents the probability, for each vacancy, that it will visit candidate $n$, as a function of candidate $n$ 's reserve wage $r_{n}$ and the reserve wages for all other candidates, $r$. Note that $p_{n}\left(r_{n}, r\right)$ is decreasing in $r_{n}$, so the higher the reserve wage posted by candidate $n$, the less likely a vacancy is to approach that candidate.

We now analyse this choice of reserve wages.

\subsection{Candidates' Reserve Wage Choices}

Given the policy parameters $(\theta, \sigma, \tau, \omega)$ and the number of vacancies $M$, candidates choose their reserve wages to maximize their expected payoffs in a simultaneous move game with other candidates. Let $q_{n 0}$ and $q_{n 1}$ denote the probabilities that candidate $n$ will receive zero offers $\left(m_{n}=0\right)$ and exactly one offer $\left(m_{n}=1\right)$ respectively. Thus: 


$$
q_{n 0}\left(r_{n}, r\right)=\left(1-p_{n}\left(r_{n}, r\right)\right)^{M}
$$

and

$$
q_{n 1}\left(r_{n}, r\right)=M p_{n}\left(r_{n}, r\right)\left(1-p_{n}\left(r_{n}, r\right)\right)^{M-1}
$$

Where $p_{n}\left(r_{n}, r\right)$ is given in equation (1.4). The after-tax expected payoff function for candidate $n$ is therefore given by:

$$
\begin{gathered}
V_{n}\left(r_{n}, r\right)=q_{n 0}\left(r_{n}, r\right) \min \{\theta(1-\tau)+\tau \omega, \theta\}+q_{n 1}\left(r_{n}, r\right) \min \left\{r_{n}(1-\tau)+\tau \omega, r_{n}\right\} \\
+\left(1-q_{n 0}\left(r_{n}, r\right)-q_{n 1}\left(r_{n}, r\right)\right)((y+\sigma)(1-\tau)+\tau \omega)
\end{gathered}
$$

Since candidates choose their reserve wages simultaneously, an equilibrium array of reserve wages is found by a standard Nash argument:

$$
r_{n}^{*}=\arg \max _{r_{n}} V_{n}\left(r_{n}, r^{*}\right)
$$

Proposition 1. The unique symmetric equilibrium reserve wage is:

$$
r_{n}^{*}=r^{*}=\frac{(M-1)((y+\sigma)(1-\tau)+\tau \omega)+(N-1)^{2} \min \{\theta(1-\tau)+\tau \omega, \theta\}}{(M-1)+(N-1)^{2}}
$$

Proof: In the Appendix.

Equation (1.6) shows that the symmetric equilibrium reserve wage is a weighted average of the after-tax wage that the candidate would receive if more than one vacancy approached him $((y+\sigma)(1-\tau)+\tau \omega)$ and the candidate's outside option $(\min \{\theta(1-\tau)+\tau \omega, \theta\})$. Also, substituting $r_{n}^{*}=r^{*}$ into equation (1.4), we derive the result that, in the equilibrium, all vacancies assign equal probability to visiting each candidate: 


$$
p_{n}^{*}\left(r^{*}, r^{*}\right)=p^{*}=1 / N
$$

Let $q_{0}{ }^{*}$ and $q_{1}{ }^{*}$ denote the probabilities that, in the symmetric equilibrium, a candidate will receive zero offers and exactly one offer respectively. Thus:

$$
q_{0}^{*}=\left(1-p^{*}\right)^{M}
$$

and

$$
q_{1}^{*}=M p^{*}\left(1-p^{*}\right)^{M-1}
$$

We can now calculate the expected number of matches $(x)$ in equilibrium. Since $q_{0}{ }^{*}$ is the probability of a candidate receiving no offers, $1-q_{0}{ }^{*}$ is the probability the candidate will receive at least one offer. In the symmetric equilibrium, the expected number of matches is given by $N$ times this number. Using the fact that $p^{*}=1 / N$ :

$$
x^{*}(N, M)=N\left(1-\left(\frac{N-1}{N}\right)^{M}\right)
$$

The expected rate of equilibrium unemployment $\left(U^{*}(N, M)\right)$ is simply the expected number of candidates who receive no offers, divided be the size of the labor force (here, the size of the labor force is the same as the number of candidates), which is just $q_{0}{ }^{*}$. Letting $p^{*}=1 / N$, we get:

$$
U^{*}(N, M)=\left(\frac{N-1}{N}\right)^{M}
$$

Notice that none of the policy parameters $(\theta, \sigma, \tau, \omega)$ appear in equations (1.8) or (1.9). This leads to the next proposition. 
Proposition 2: $\quad$ For any given number of vacancies $M$, in the symmetric equilibrium, the number of matches and the number of unemployed are independent of the government policy parameters.

Proof: $\quad$ Clear from inspection of equations (1.8) and (1.9).

The intuition for this result is quite straightforward. In the symmetric equilibrium, all vacancies announce the same reserve wage $r^{*}$ (given in equation (1.6)), and so all vacancies assign the same probability of visiting to each candidate. Hence, matching occurs through the simple urn-ball process summarized in equations (1.8) and (1.9). As long as all candidates are identical in this way, this matching process will be obtained in equilibrium. ${ }^{7}$ As we show below, the policy parameters can affect vacancy profits, and hence affect the number of vacancies that enter; but, for any given number of entrants, these parameters will play no further role on matching or unemployment.

\subsection{The Entry of Vacancies}

Given the policy parameters $(\theta, \sigma, \tau, \omega)$, firms choose how many vacancies to create. The substitution of equations (1.6) and (1.7) into equation (1.3) provides an expression for the expected payoff for a vacancy in the symmetric equilibrium, given that the vacancy has already been created:

$$
\Pi^{*}\left(r^{*}\right)=\left(1-\frac{1}{N}\right)^{M-1}\left(y+\sigma-r^{*}\right)
$$

The cost of entry is given by the parameter $k$. The free entry condition is:

$$
\Pi^{*}\left(r^{*}\right)-k=0
$$

\footnotetext{
${ }^{7}$ Adding worker heterogeneity does not change the fact that a symmetric mixed strategy equilibrium exists, but does break the result that equal probability will be assigned to each worker in that equilibrium. In general, higher probability will be assigned to more productive workers. See Julien, Kennes, and King (2005a) for models of this type, without policy parameters.
} 
Together, equations (1.10) and (1.11) imply:

$$
\left(\frac{N-1}{N}\right)^{M-1}\left(y+\sigma-r^{*}\right)=k
$$

Equations (1.6) and (1.12) are two equations in two unknowns, $M$ and $r^{*}$, that simultaneously determine the number of vacancies that enter and the reserve wage in the symmetric equilibrium.

To simplify the analysis, for most of the remainder of the paper, we impose the following restriction on the relative values of non-taxable income and unemployment benefits: $\omega \leq \theta$ (and, therefore, also $\omega \leq r_{n}$ since $\theta \leq r_{n}$ by assumption).

This simplifies equations (1.1), (1.5), and (1.6) to:

$$
\begin{gathered}
b\left(r_{n}, m_{n}\right)=\left\{\begin{array}{cc}
\theta(1-\tau)+\tau \omega & \text { if } m_{n}=0 \\
r_{n}(1-\tau)+\tau \omega & \text { if } m_{n}=1 \\
(y+\sigma)(1-\tau)+\tau \omega & \text { if } m_{n}>1
\end{array}\right. \\
V_{n}\left(r_{n}, r\right)=q_{n 0}\left(r_{n}, r\right)(\theta(1-\tau)+\tau \omega)+q_{n 1}\left(r_{n}, r\right)\left(r_{n}(1-\tau)+\tau \omega\right) \\
+\left(1-q_{n 0}\left(r_{n}, r\right)-q_{n 1}\left(r_{n}, r\right)\right)((y+\sigma)(1-\tau)+\tau \omega)
\end{gathered}
$$

Re-arranging (1.12) we also get:

$$
r^{*}=y+\sigma-k\left(\frac{N}{N-1}\right)^{M-1}
$$


Equating (1.6') and (1.12') we obtain the following single equation determining the equilibrium number of vacancies $M$ :

$$
y+\sigma-k\left(\frac{N}{N-1}\right)^{M-1}=\frac{(M-1)((y+\sigma)(1-\tau)+\tau \omega)+(N-1)^{2}(\theta(1-\tau)+\tau \omega)}{(M-1)+(N-1)^{2}}
$$

where $M>1$ is constrained to be an integer. We can define $M$ as the integer which minimizes the difference between the left hand side and the right hand side of equation (1.13). Once the integer value of $M$ has been determined from the above equation, the reservation wage $r_{n}^{*}=r^{*}$ is given by equation (1.6'). ${ }^{8}$

Given the set of policy parameters $(\theta, \sigma, \tau, \omega)$, this completes the solution of the model.

Section 3 provides the comparative static properties of the symmetric equilibrium.

\subsection{The Government's Budget Constraint}

In the symmetric equilibrium, the government's budget constraint per candidate is:

$$
\theta q_{0}^{*}+\sigma\left(1-q_{0}^{*}\right)=\tau\left((\theta-\omega) q_{0}{ }^{*}+\left(r^{*}-\omega\right) q_{1}^{*}+(y+\sigma-\omega)\left(1-q_{0}{ }^{*}-q_{1}^{*}\right)\right)
$$

Substituting in $p^{*}=1 / N$, we get:

$$
\begin{aligned}
& \theta\left(\frac{N-1}{N}\right)^{M}+\sigma\left(1-\left(\frac{N-1}{N}\right)^{M}\right)= \\
& \tau\left((\theta-\omega)\left(\frac{N-1}{N}\right)^{M}+\left(r^{*}-\omega\right) \frac{M}{N}\left(\frac{N-1}{N}\right)^{M-1}+(y+\sigma-\omega)\left(1-\left(\frac{N-1}{N}\right)^{M}-\frac{M}{N}\left(\frac{N-1}{N}\right)^{M-1}\right)\right)
\end{aligned}
$$

Where $r^{*}$ and $M$ are determined by equations (1.6') and (1.13).

\footnotetext{
${ }^{8}$ Note that this means that equation (1.12') will not hold exactly for the integer M.
} 


\section{THE APPROXIMATE (LIMIT) ECONOMY}

We now consider an economy that approximates the economy presented in Section 1, taken at the limit where the number of candidates is infinite. We consider three different cases. First, the economy with arbitrary values of the government policy parameters. Second, the economy with no government policy parameters. Thirdly, we consider the economy with government policy parameters set to values that are consistent with both ex ante efficiency and ex post equity.

To analyze the limit economy, in all cases, we start by defining labour market tightness as $\phi=M / N$.

\subsection{The Limit Economy with Arbitrary Government Policy Parameters}

The limit economy is considerably simpler to solve and analyse than the finite economy of Section 1 above. In particular, unlike the finite economy, the limit economy has simple closed-form solutions for the values of the key endogenous variables in the symmetric equilibrium.

Substitution of $M=\phi N$ into equations (1.6') and (1.7), for any $\phi$, and taking the limit as $N \rightarrow \infty$, one obtains: ${ }^{9}$

$$
r^{*}=\theta(1-\tau)+\tau \omega
$$

$$
p^{*}=0
$$

Consider equation (2.7) first. This shows the straightforward result that, as the number of candidates gets large, given that the vacancies assign equal probability to approaching

\footnotetext{
${ }^{9}$ The derivation of (2.6') uses L'Hôpital's rule.
} 
each candidate, the probability that any one employer will approach any particular candidate goes to zero.

The reasoning behind equation (2.6') is less straightforward, but quite important. In this limit, workers' reserve wage announcements are driven down to their outside option $(\theta(1-\tau)+\tau \omega)$. This result follows from the particular sequence of events assumed here (i.e., candidates move first in this game, by choosing their reserve wages) and the implicit assumption that candidates apply, costlessly to all vacancies, ${ }^{10}$ while vacancies are restricted to making offers to only one candidate. With randomization, vacancies place less weight on approaching each candidate than each candidate places on approaching each vacancy. (Each candidate approaches each vacancy with certainty, but each vacancy approaches any candidate with some probability less than one.) Thus, as the market size increases, keeping the ratio of vacancies to candidates constant, there are asymmetric influences of increasing $M$ and $N$ : for each candidate, the probability of being approached erodes more quickly (as $N$ increases) than the analogous probability for vacancies (as $M$ increases). This then erodes the first-mover advantage that candidates enjoy when announcing their reserve wages - driving them down to the outside option. ${ }^{11}$

Using the fact that, for any $z \in \mathbb{R}, \lim _{M \rightarrow \infty}(1+z / M)^{M}=e^{z}$ (where $z=-M / N=-\phi$ here), the equilibrium matching function and unemployment rate in the limit economy become, respectively:

$$
\begin{gathered}
x(N, \phi)=N\left(1-e^{-\phi}\right) \\
U^{*}(\phi)=e^{-\phi}
\end{gathered}
$$

\footnotetext{
${ }^{10}$ This assumption is made explicit, and examined carefully, in Albrecht, Gautier, and Vroman (2006).

${ }^{11}$ Equation (2.6') is also consistent with Theorem 2 in McAfee (1993). In the absence of any government policy parameters, the limit economy in this paper is a special case of the economy studied in McAfee's paper. (McAfee considers only a limit economy.)
} 
Similarly, the entry condition for vacancies (1.12) becomes:

$$
e^{-\phi}\left(y+\sigma-r^{*}\right)=k
$$

The government's budget constraint becomes:

$$
(\theta(1-\tau)+\tau \omega) e^{-\phi}+\sigma\left(1-e^{-\phi}\right)=\tau\left(\left(r^{*}-\omega\right) \phi e^{-\phi}+(y+\sigma-\omega)\left(1-e^{-\phi}-\phi e^{\phi}\right)\right)
$$

Recall that, in the finite economy, $r^{*}$ and $M$ are determined simultaneously in equations (1.6) and (1.12). In the limit economy equation (2.6') determines $r^{*}$ independently of $M$. Substitution of (2.6') into (2.12) yields:

$$
e^{-\phi}=\frac{k}{y+\sigma-\theta(1-\tau)-\tau \omega}
$$

Equation (2.14) determines the equilibrium value of $\phi$ in this model. Taking the logarithm of both sides of this equation yields the symmetric equilibrium value of labour market tightness:

$$
\phi^{*}=\ln (y+\sigma-\theta(1-\tau)-\tau \omega)-\ln k
$$

Equilibrium vacancies $M^{*}=\phi^{*} N$ are therefore:

$$
M^{*}=[\ln (y+\sigma-\theta(1-\tau)-\tau \omega)-\ln k] N
$$

Also, substitution of (2.14) into (2.9) yields the symmetric equilibrium unemployment rate:

$$
U^{*}=\frac{k}{y+\sigma-\theta(1-\tau)-\tau \omega}
$$


Notice that, in general, both the number of vacancies and the unemployment rate are functions of all the government policy parameters $(\theta, \sigma, \tau, \omega)$.

Section 3 provides the comparative static properties of the symmetric equilibrium.

\subsection{The Limit Economy with No Government}

It is instructive, at this point, to consider the special case when all the government policy parameters are set equal to zero. The key equations (2.6'), (2.14) and (2.16) become, respectively:

$$
\begin{gathered}
r^{*}=0 \\
e^{-\phi^{*}} y=k
\end{gathered}
$$

or equivalently,

$$
\phi^{*}=\ln y-\ln k
$$

$$
U^{*}=\frac{k}{y}
$$

Equation (2.6") shows that, in this case, absence of any other income, candidates' outside option is simply zero. Equation (2.12) shows the vacancy entry condition in this case. On the left side is a vacancy's ex ante expected revenue after wages have been paid. Due to the auction structure, a vacancy will receive positive revenue only if the vacancy is alone when approaching the candidate (as always, if more than one vacancy approaches the candidate, the revenue is bid away). The probability of this occuring is $e^{-\phi}$. When vacancies are alone in approaching a candidate, their revenues are $y$ and they pay the worker the outside option, which is zero. Thus, the left hand side of (2.12') presents vacancies' expected revenues after wages have been paid. The right hand side is the cost of the vacancy. Recognizing that $e^{-\phi}$ is also the unemployment rate, equation (2.16') 
then presents a very natural condition for equilibrium unemployment: this is given by the ratio of the cost of a vacancy to the output produced by the vacancy when filled. ${ }^{12}$

\section{COMPARATIVE STATICS}

Qualitatively, the comparative statics for the limit economy and the finite economy turn out to be almost identical. Given our assumption that $\omega \leq \theta$, the only difference between the limit and finite economies is in the effect of the tax rate $\tau$ in the case where $\omega=\theta$.

Proposition 3: $\quad$ In the symmetric equilibrium of both the finite and limit economies, assuming that $\tau \in(0,1)$ :

a) Unemployment benefits $\theta$ decrease vacancies and increase unemployment.

b) Employment subsidies $\sigma$ increase vacancies and decrease unemployment.

c) The non-taxable income level $\omega$ decreases vacancies and increases unemployment.

d) For the finite economy, $\tau$ increases vacancies and decreases unemployment. For the limit economy, the effect of the income tax rate $\tau$ depends on the relative values of $\omega$ and $\theta$. If $\omega<\theta$ then $\tau$ increases vacancies and decreases unemployment. If $\omega=\theta$ then income taxes have no effect on vacancies or unemployment.

Proof: $\quad$ For the limit economy, these results follow readily from the evaluation of partial derivatives of equations (2.15) and (2.16). For the finite economy, proofs are provided in the appendix.

If we relax our assumption that $\omega \leq \theta$, the comparative static results (a) - (c) still hold. In the limit economy, if $\omega>\theta$ (i.e., unemployment benefits are not taxable) then $\tau$

\footnotetext{
${ }^{12}$ A similar interpretation holds, more generally, in the model with general policy parameter values. In this case, in equation (2.14), $y$ is replaced by $y+\sigma-\theta(1-\tau)-\tau \omega$, which is the total surplus generated by and employment relationship. We thank a referee for pointing this out.
} 
decreases vacancies and increases unemployment. In the finite economy, the direction of the effect of the tax rate $\tau$ on vacancies and unemployment cannot be determined if $\omega>\theta$.

To understand the effects that these policy variables have on unemployment, it is important to keep in mind the results in Proposition 2: for any given number of vacancies $M$, the unemployment rate is independent of any of the policy parameters. Thus, the changes in the unemployment rate identified in Proposition 3 occur purely through the channel of altered vacancy creation.

Unemployment benefits affect the number of vacancies and the unemployment rate through their effect on candidates' outside options, and thereby the reserve wage $r^{*}$. An increase in benefits raises the value of candidates' outside options (the reserve wage). Through the auction mechanism, this raises the wage received by candidates that have only one vacancy approach them and leaves unaffected the wage received by the candidates that have more than one vacancy approach them. This raises average wages, for any given number of vacancies, that entering vacancies can expect to pay. This lowers the expected payoff for each vacancy and thus reduces the number of vacancies created in equilibrium. Through the matching process, this reduction in vacancies then increases the unemployment rate.

Employment subsidies do not affect candidate's outside options or the reserve wage $r^{*}$, but do increase the payoff for vacancies that are alone when they approach a worker. (Vacancies that are not alone have the value of subsidy bid away by Bertrand competition, so the value of $\sigma$ does not affect firm payoffs in this case.) An increase in $\sigma$ will therefore increase the ex ante expected payoff for vacancies, for any given $M$, and thereby increase vacancy creation. Through the matching process, this increase in the number of vacancies decreases the unemployment rate.

The non-taxable income level $\omega$ influences candidates' outside options through its effect on after-tax benefit payments. An increase in $\omega$ will increase these after-tax payments 
and thereby increase the value of candidates' outside options. (For example, in the limit of the range considered here, where $\omega=\theta$, candidates receive the full value of the benefits.) The effects, then, are similar to the effect produced by increasing the level of benefits: the reserve wage (and, thereby, wages for candidates who are approached by only one vacancy) rises, this raises the average wages that vacancies can expect to pay, for any given number of vacancies. This lowers the expected payoff for each vacancy and thus reduces the number of vacancies created in equilibrium. Through the matching process, this reduction in vacancies then increases the unemployment rate.

Changes in the income tax rate $\tau$ also influence vacancies and unemployment through the channel of changing candidates' outside options. In the finite economy, an increase in the income tax rate increases vacancies and decreases unemployment. In the limit economy, this influence is felt only in the presence of benefit payments, and only if the non-taxable income level $\omega$ is strictly less than the benefit payments $\theta$. If $\omega=\theta$ then benefits are entirely untaxed, and changes in the tax rate have no effect on the value of candidates' outside option, which is simply $\theta$ in this case.

In the finite economy, and in the limit economy when $\omega<\theta$, an increase in the income tax will reduce candidates' after-tax outside option. This, then, will have the opposite effect of an increase in $\omega$ discussed above: the reserve wage (and, thereby, wages for candidates who are approached by only one vacancy) falls, which reduces the average wages that vacancies can expect to pay, for any given number of vacancies. This increases the expected payoff for each vacancy and thus increases the number of vacancies created in equilibrium. Through the matching process, this increase in vacancies then decreases the unemployment rate. 


\section{OPTIMAL GOVERNMENT POLICY}

\subsection{Ex Ante Constrained-Efficient Allocations in the Limit Economy}

Consider, first, a planner that is somehow able to control the entry of vacancies, but still faces the same coordination friction as the private agents. With this direct control, the planner then chooses $\phi$ to maximize total expected surplus:

$$
S=N\left(\left(1-e^{-\phi}\right) y-\phi k\right)
$$

The first order condition from this maximization problem is:

$$
e^{-\phi} y=k
$$

or equivalently,

$$
\phi=\ln (y / k)
$$

Which is identical to condition (2.12') above which determines the symmetric equilibrium value of $\varphi$ in the limit economy with no government. ${ }^{13}$ This illustrates the (now well-known) result that, in the absence of any distortionary structures, the decentralized equilibrium of large directed search economies is constrained-efficient. Thus, given that agents are risk neutral, setting all the policy variables equal to zero is an optimal government policy in this framework, from an ex ante point of view.

\subsection{Ex Ante Constrained-Efficient Allocations in the Finite Economy}

As in the limit economy, consider a planner that is somehow able to control the entry of vacancies, but still faces the same coordination friction as the private agents. With this direct control, the planner then chooses the number of vacancies $M$ to maximize total expected surplus:

\footnotetext{
${ }^{13}$ The concavity of the surplus function ensures that the first order condition identifies a global maximum.
} 


$$
S=N\left(1-\left(\frac{N-1}{N}\right)^{M}\right) y-M k
$$

The first order condition from this maximization problem is:

$$
-N y\left(\frac{N-1}{N}\right)^{M} \ln \left(\frac{N-1}{N}\right)=k
$$

This equation determines the optimal number of vacancies $M^{* *}$, which is given by:

$$
M^{* *}=\frac{\ln (y / k)+\ln N\left(\ln \left(\frac{N}{N-1}\right)\right)}{\ln \left(\frac{N}{N-1}\right)}
$$

where $M^{* *}$ is restricted to be an integer.

To find the decentralised equilibrium $M^{*}$ in the finite economy with no government, we set all policy parameters equal to zero in equation (1.13) and re-arrange to get:

$$
\frac{k}{y}\left(\frac{N}{N-1}\right)^{M^{*}}=\frac{N(N-1)}{\left(M^{*}-1\right)+(N-1)^{2}}
$$

We can re-arrange the first order condition for the social optimum $M^{* *}$ as follows:

$$
\frac{k}{y}\left(\frac{N}{N-1}\right)^{M^{*}}=N \ln \left(\frac{N}{N-1}\right)
$$

We have "constrained efficiency" in the finite economy, namely $M^{*}=M^{* *}$, if and only if the right hand sides of the above equations are equal: 


$$
\ln \left(\frac{N}{N-1}\right)=\frac{(N-1)}{\left(M^{*}-1\right)+(N-1)^{2}}
$$

Using the fact that, even for relatively small values of $N$ (say $N \geq 10$ ),

$$
\ln \left(\frac{N}{N-1}\right) \approx \frac{2}{2 N-1}
$$

It can be shown that $M^{*}=M^{* *}$ if and only if:

$$
\frac{M^{*}-1}{N-1}=\frac{1}{2}
$$

So "constrained efficiency" holds in the finite economy only on the above condition. In this special case, the social optimum and the equilibrium coincide $\left(M^{*}=M^{* *}\right)$ regardless of whether $N$ is large. Of course, for arbitrary values of $y, k$ and $N$, this will not, in general, be the case.

If $N$ is sufficiently large, however, the social optimum $\phi^{* *}$ and the equilibrium $\phi^{*}$ are very close to being identical in the finite economy.

From above, the social optimum $\phi^{* *}$ is given by:

$$
\phi^{* *}=\frac{\ln (y / k)+\ln \left(N \ln \left(\frac{N}{N-1}\right)\right)}{N \ln \left(\frac{N}{N-1}\right)}
$$

Now, if we take the limit as $N \rightarrow \infty$, we have:

$$
\lim _{N \rightarrow \infty} \phi^{* *}=\ln (y / k)
$$


Therefore, in the limit as $N \rightarrow \infty$, the socially optimal value $\phi^{* *}$ is exactly the same as the optimal value of $\phi$ in the limit economy, which is in turn identical to the equilibrium value $\phi^{*}$ in the limit economy with no government.

Further, in the limit as $N \rightarrow \infty$, the equilibrium $\phi^{*}$ in the finite economy is the same as the equilibrium of the limit economy, namely $\phi^{*}=\ln (y / k)$ when policy parameters are set to zero. ${ }^{14}$

So in the limit as $N \rightarrow \infty$, setting all policy parameters equal to zero results in $\phi^{* *}=\phi^{*}$. That is, the equilibrium $\phi^{*}$ coincides with the socially optimal value $\phi^{* *}$. Thus, from an ex ante point of view, zero government intervention is an approximately optimal policy in the finite economy provided that $N$ is sufficiently large.

In Section 5, we conduct numerical simulations to examine how large $N$ must be in order for this approximation to be close.

\subsection{Ex Ante Constrained Efficiency and Ex Post Equity}

The policy of setting government parameters equal to zero exposes candidates to the full income risk associated with the random matching process. While it is true that candidates are risk neutral in this economy, it is still natural to question whether or not an alternative policy setting may exist which preserves the constrained efficiency of the zero policy setting, but also eliminates the income risk. This question is answered in the following proposition, for the limit economy.

\footnotetext{
${ }^{14}$ This follows immediately from the way that the infinite economy equilibrium values $r^{*}$ and $\phi^{*}$ were determined by taking the limits of equations (1.6') and (1.12) as $N \rightarrow \infty$.
} 
Proposition 4: $\quad$ In the symmetric equilibrium of the limit economy, the following policy settings maximize expected surplus, eliminate income risk for candidates, and satisfy the government's budget constraint:

$$
\sigma=\omega=\theta=y\left(1-q_{0}^{*}-q_{1}^{*}\right)=y\left(1-e^{-\varphi}-\varphi e^{-\varphi}\right) \text { and } \tau=1
$$

Proof: Setting $\sigma=\omega=\theta$ and $\tau=1$ in equation (2.14) yields equation (4.2), and in equation (2.6') yields $r^{*}=\theta$. Using these in the government budget constraint (2.13) yields the after-tax payment to unemployed workers $\theta=y\left(1-e^{-\phi}-\phi e^{-\phi}\right)$. From equation (1.1'), workers who receive a visit from one vacancy obtain wage $r^{*}(1-\tau)+\tau \omega$ which, using the above values becomes $\omega=\theta=y\left(1-e^{-\phi}-\phi e^{-\phi}\right)$. Also from equation (1.1'), workers who receive visits from more than one vacancy obtain the wage $(y+\sigma)(1-\tau)+\tau \omega$ which, using the above values, becomes $\omega=\theta=y\left(1-e^{-\phi}-\phi e^{-\phi}\right)$.

A similar result is approximately true for the finite economy. If we let $\sigma=\omega=\theta=y\left(1-q_{0}{ }^{*}-q_{1}^{*}\right)$ and $\tau=1$, we get $r^{*}=\theta$ using equation (1.6'). The government budget constraint is satisfied and income risk is eliminated, as in the limit economy. The decentralised equilibrium number of vacancies $M^{*}$ is not exactly equal to the optimal number of vacancies $M^{* *}$; however, the difference between them is very small. The difference $M^{*}-M^{* *}$ for $M^{*}, M^{* *} \in \mathbb{R}$ is approximately 0.5 for $N \geq 10$, so we get either $M^{*}-M^{* *}=1$ or $M^{*}=M^{* *}$, depending on the rounding, for the integer values $M^{*}, M^{* *} \in \mathbb{N}$. So for any $N \geq 10$, this policy setting is very close to the optimal policy. Of course, as $N \rightarrow \infty$ we get $\phi^{* *}=\phi^{*}$, hence for large values of $N$ this policy setting does maximise total expected surplus.

This policy configuration, for all its virtues, is quite drastic. The tax rate, above the critical income of $\omega$, is set at $100 \%$. The value of $\omega$ is set equal to the value of unemployment benefits. Thus, after-tax, all workers receive the same income as the unemployed. Since candidates' outside options are driven up to this same value, to re- 
establish efficient vacancy entry, the employment subsidy to firms must be equal to precisely the same amount. The government budget constraint then determines what that amount is. The starkness of this optimal configuration is due largely to the fact that all workers are homogeneous in this model so any income disparity reflects only the luck of the draw - whose influence insurance is designed to eliminate. A model where workers invest in skills at a prior stage would alter this result to some degree. ${ }^{15}$

\section{THE ACCURACY OF THE APPROXIMATION}

To get a sense of how quickly the exact economy converges to its limit as the size of the economy increases, we simulated the model, for particular values of the parameters, and for a range of values of $N$.

We set all of the policy parameters equal to zero. As shown in Section 4.1, in this case, the equilibrium and efficient allocations coincide in the limit economy. Moreover, this allocation also coincides with the equilibrium allocation under the optimal policy configuration identified in Proposition 4.

In the finite economy, however, the efficient $\phi^{* *}$ and the equilibrium $\phi^{*}$ are not the same. We compare the equilibrium value $\phi^{*}$ in the finite economy to both the social optimum $\phi^{* *}$ for the finite economy and the limit economy value of $\phi^{*}\left(=\phi^{* *}\right)$.

In the absence of policy parameters, the only remaining parameters in the model are $y$ and k. As ballpark figures, for weekly incomes, we set: ${ }^{16}$

$$
y=500 \quad k=50 .
$$

${ }^{15}$ See Julien, Kennes, and King (2006a) for one such model.

${ }^{16}$ For a calibration of the dynamic version of the limit economy, see Julien, Kennes, and King (2005b). 
Using equations (2.6”), (2.12') and (2.16'), respectively, from section 2.2 above, we find the following numbers for the symmetric equilibrium reserve wage, market tightness, and unemployment in the limit economy:

$$
r^{*}=0 \quad \phi^{*}=2.3026 \quad U^{*}=0.1
$$

Figure 1 plots the values of equilibrium market tightness $\phi^{*}$ from the finite economy, for $N=2, \ldots, 100$, with the policy variables set at zero, using the integer value of $M^{*}$ determined by solving (1.13):

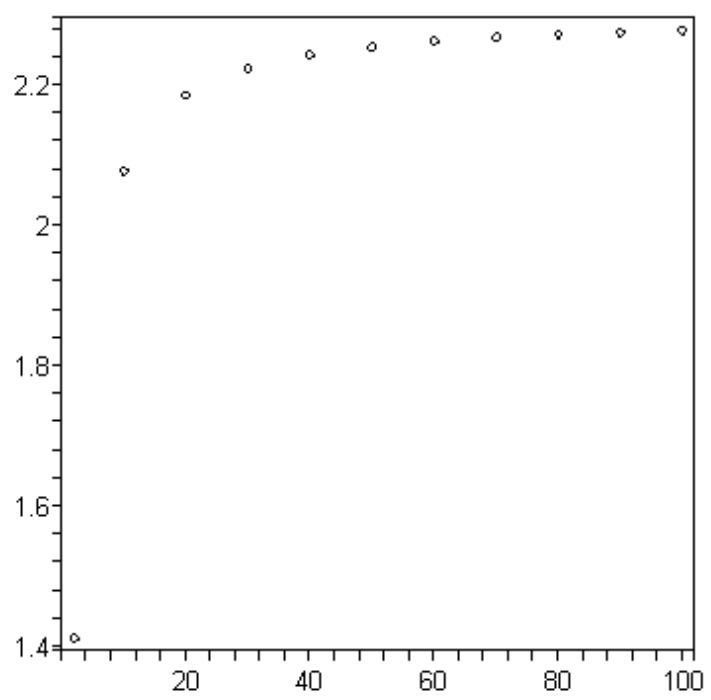

Figure 1: Market Tightness in the Finite Economy

When $N=2, \phi^{*}=1.5$, only $65 \%$ of the limit value of $\phi^{*}$ and $75 \%$ of the efficient value of $\phi^{* *}=2.0$. Once $N$ increases to $10, \phi^{*}=2.1$, approximately $91 \%$ of the limit value and $96 \%$ of the efficient value of $\phi^{* *}=2.2$. At $N=50, \phi^{*}=2.26$, or $98 \%$ of the limit value and $99 \%$ of the efficient value of $\phi^{* *}=2.28$. At $N=100, \phi^{*}=2.28$, or $99 \%$ of both the limit value and the efficient value of $\phi^{* *}=2.30$. At $N=200, \phi^{*}=2.29$, or $99.5 \%$ of the limit value and $99.6 \%$ of the efficient value of $\phi^{* *}=2.30$. Thus, market tightness 
converges quite quickly to both the limit value and the efficient value (which converge to each other).

Figure 2 plots the values of the equilibrium unemployment rate $U^{*}$ from the finite economy, for $N=2, \ldots, 100$, using equation (1.9), once the integer value of $M^{*}$ has been determined from (1.13):

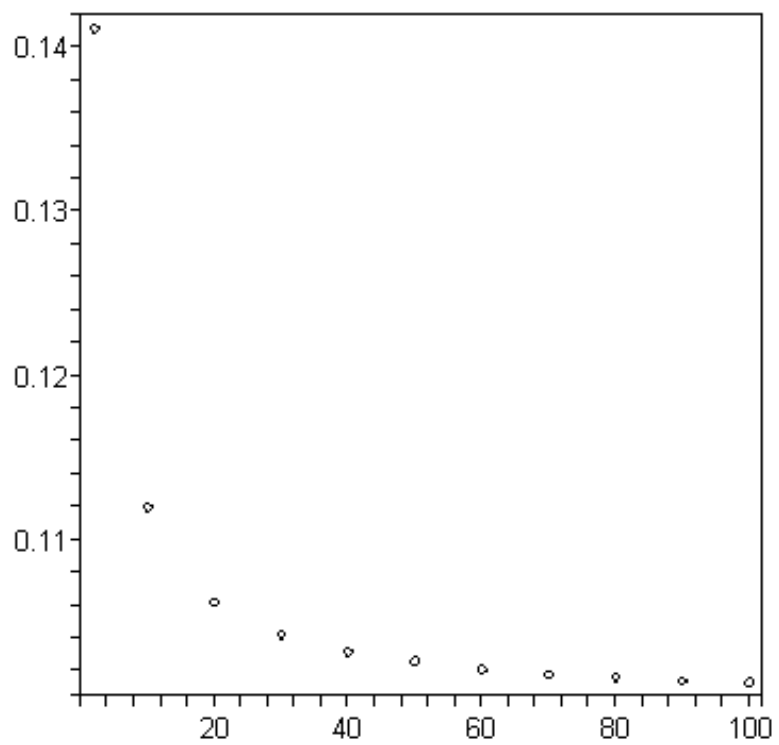

Figure 2: The Unemployment Rate in the Finite Economy

When $N=2, U^{*}=0.1250$, or $125 \%$ of the limit value of $U^{*}$ and $200 \%$ of the efficient value of $U^{* *}=0.0625$ obtained using the efficient market tightness ratio $\phi^{* *}$. Once $N$ increases to $10, U^{*}=0.1094$, or $109 \%$ of the limit value and $111 \%$ of the efficient value $U^{* *}=0.0985$. At $N=50, U^{*}=0.1020$, or $102 \%$ of the limit value and $102 \%$ of the efficient value $U^{* *}=0.0999$. At this stage, the efficient unemployment value for the finite economy is very close to the limit value. At $N=100, U^{*}=0.1011$, or $101.1 \%$ of the limit value. At $N=200, U^{*}=0.1007$, or $100.7 \%$ of the limit value. Thus, unemployment converges quite quickly. 
Figure 3 plots the values of $r^{*}$ from the finite economy, for $N=2, \ldots, 100$, with the policy parameters set to zero, using equation (1.6') once the integer value of $M^{*}$ has been determined from (1.13):

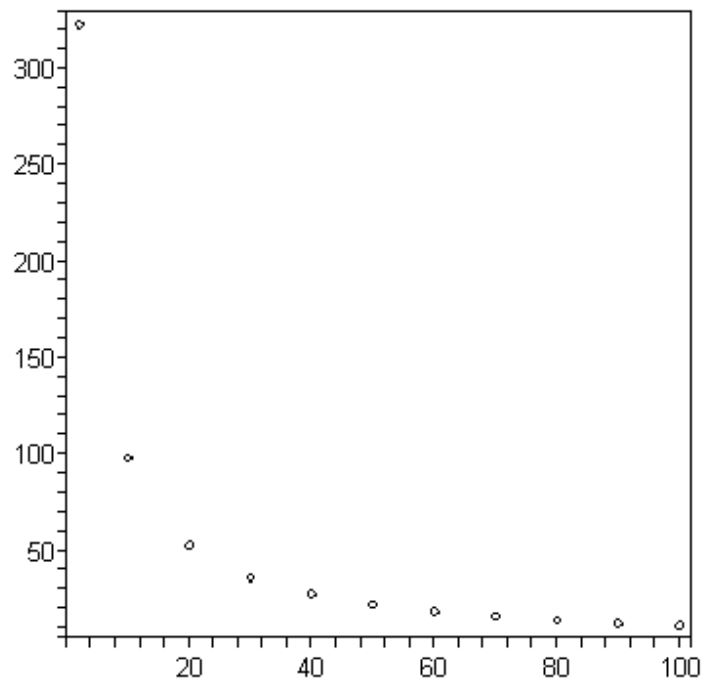

Figure 3: Reserve Wages in the Finite Economy

For small values of $N$, the reserve wage in the exact economy is quite high relative to the value of $y$. In the smallest case, where $N=2, r^{*}=333.33$, which is approximately $60 \%$ of output. ${ }^{17}$ The reserve wage in the exact economy drops relatively quickly as $N$ increases, but is still fairly significant, even for some relatively large values of $N$. For example, when $N=20: r^{*}=53.22$, or approximately $10.6 \%$ of $y$. When $N=100$, $r^{*}=11.32$, or $2.3 \%$ of $y$. Even at $N=200$ (not shown in Figure 3 ) $r^{*}$ is not negligible: 5.70 , or $1.1 \%$ of $y$.

Overall, the limit economy gives a reasonably accurate approximation (within 1\% of their true values) of market tightness and unemployment in the finite economy, for any $N>100$. Similar accuracy for the reserve wage approximation requires a market of somewhat larger size: $N>200$.

\footnotetext{
${ }^{17}$ Efficient entry is induced in the finite economy when the reserve wage is equal to the worker's outside option, which, in this case is zero. This rule holds true in more general models. See Julien, Kennes, and King (2005a) for details.
} 


\section{CONCLUSION}

The comparative static effects of employment subsidies on vacancies and unemployment, in the directed search model considered here, with worker auctions, are very similar to those found, in Pissarides (1985), in models with random matching and Nash bargaining. In both modelling environments, these subsidies directly increase firm payoffs, and thus lead to increments in vacancy entry, job creation and, through the matching process, reductions in unemployment. The only significant difference is that, with directed search, the matching process is an equilibrium phenomenon, rather than a technological one. However, with homogeneous workers, the equilibrium matching process is invariant to changes in the policy parameters - so this difference plays no substantive role in assessing the comparative statics.

However, the directed search model adds another dimension that is not considered in Pissarides' model: wage dispersion. In both models, workers are homogenous, ex ante. In Pissarides' model, all workers are paid the same amount, ex post. In this model, the equilibrium wage distribution is non-degenerate: some workers (those who have been approached by only one vacancy) are paid only their reserve wage, while others (who have been approached by more than one vacancy) are paid the total value of the surplus from the match. Employment subsidies do not affect the equilibrium wages of the first type of worker, but do increase the wages of the second. Thus, in this environment, ceteris paribus, increments in employment subsidies increase wage dispersion.

Similar statements can be made about the effects of unemployment insurance. In both models, this raises expected wages, discourages entry and, thereby, increases unemployment. However, here, the difference in the chain of causation across the models is more substantive. In Pissarides' model, unemployment insurance affects workers' threat points in the Nash bargaining solution and, thus, raises wages for all workers. In this model, however, unemployment insurance affects workers' outside options, which raises the wage only for workers in low-paid jobs (i.e., workers who are approached by only one vacancy). Wages for workers in high-paid jobs (i.e., workers who are 
approached by more than one vacancy) are determined by Bertrand competition, driving their wages to the maximum possible, which is independent of unemployment insurance. Hence, ceteris paribus, increments in unemployment insurance reduce wage dispersion in this model, but not in Pissarides' model.

Unemployment benefits are untaxed, in Pissarides' model, but all output from each match is subject to taxation. The tax rate forms a wedge which, analytically, plays the same role as a reduction in the worker's bargaining parameter. This reduces wages, but also reduces after-tax surplus and profits overall - thus driving down entry and increasing unemployment. While there is no precise analogue for this tax structure in the model presented here, this roughly corresponds to the special case where $\omega=\theta$. In the limit economy, in this case, wage taxes are completely neutral for vacancy creation and unemployment. (Low paid workers are paid their reserve wage, which is equal to their outside option: equal to unemployment benefits, which are untaxed. High-paid workers always receive the total surplus - which is independent of the tax rate. Thus, firm profits are independent of the tax.) In the finite economy, low-paid workers are paid their reserve wage, which is strictly above their outside option (unemployment benefits) and, so some of their wage is taxed. An increment in the wage tax therefore reduces the reserve wage, and thereby, expected wages. This increases firm profitability, driving up vacancy creation, and reducing unemployment. However, the size of this effect can be quite small, for any significant size of the market.

Optimal policy is not considered in Pissarides' original study, but has been considered subsequently in other studies using similar models. As mentioned above, Lehmann and Linden (2007) a wage tax of $100 \%$ to overcome the hold-up problem in economies of this sort. No hold-up problem exists in the limit directed search economy that we considered here, and so it provides no efficiency justification for such a tax scheme. However, a $100 \%$ tax rate for all income greater than the unemployment benefit rate can be justified on equity grounds using this model, as long as subsidies to firms are also used completely offset the effect of unemployment benefits on workers' outside options - thereby preserving efficiency. 
The starkness of this policy points to ways in which this model could be fruitfully extended: including worker effort and skill accumulation, or heterogeneity on the other side of the market Worker homogeneity is a key feature of this model, which is responsible for delivering some of its more stark results. Relaxing this assumption, particularly by allowing for endogenous heterogeneity, would be an interesting extension. Firstly, the equilibrium matching process is a function of worker heterogeneity in this type of model. ${ }^{18}$ Thus, if this heterogeneity responds to government policy (as one should expect that it would) then government policy would affect the matching process itself something that does not happen in the current model. The policy prescription of a $100 \%$ tax rate above the unemployment benefit level would also likely change with endogenous heterogeneity. In the current model, luck is the only source of income dispersion, and there are no incentive effects from this tax scheme. However, in a model where workers invest in skills, this would no longer be the case. ${ }^{19}$ In that framework, in the absence of skill-specific tax rates, we should expect trade-offs to exist between efficiency and equity.

\footnotetext{
${ }^{18}$ See, for example, Julien, Kennes, and King (2005a).

${ }^{19}$ A very simple model of this sort is considered in Julien, Kennes, and King (2006a), but with government policy variables.
} 


\section{APPENDIX}

Proof of Proposition 1:

All workers $n=1, \ldots, N$ simultaneously solve the following problem:

$$
\max _{r_{n}} V_{n}\left(r_{n}, r^{*}\right)
$$

The first-order conditions simplifies to

$$
q_{n 1}\left(r_{n}, r^{*}\right)\left(1+\frac{\Phi}{p_{n}\left(r_{n}, r^{*}\right)\left(1-p_{n}\left(r_{n}, r^{*}\right)\right)} \frac{\partial p_{n}\left(r_{n}, r^{*}\right)}{\partial r_{n}}\right)=0, \quad \forall n=1, \ldots, N
$$

where $q_{n 1}\left(r_{n}, r^{*}\right)$ is defined previously and

$\Phi=(M-1) p_{n}\left(r_{n}, r^{*}\right)\left((y+\sigma)(1-\tau)+\tau \omega-r_{n}\right)+\left(1-p_{n}\left(r_{n}, r^{*}\right)\right)\left(r_{n}-\min \{\theta(1-\tau)+\tau \omega, \theta\}\right)$.

Substituting

$$
\begin{aligned}
& \frac{\partial p_{n}\left(r_{n}, r^{*}\right)}{\partial r_{n}} \\
& =-\frac{(N-1)\left((y+\sigma)(1-\tau)+\tau \omega-r_{n}\right)^{1 /(M-1)-1}\left(1-p_{n}\left(r_{n}, r^{*}\right)\right)}{(M-1)\left[\left((y+\sigma)(1-\tau)+\tau \omega-r^{*}\right)^{1 /(M-1)}+(N-1)\left((y+\sigma)(1-\tau)+\tau \omega-r_{n}\right)^{1 /(M-1)}\right]}
\end{aligned}
$$

into the first-order condition and exploiting symmetry setting $r_{n}=r^{*}$ and $p_{n}\left(r_{n}, r^{*}\right)=1 / N, \forall n$, yields equation (1.6).

Uniqueness is shown by demonstrating that $V_{n}\left(r_{n}, r^{*}\right)$ is strictly concave in $r_{n}$ over $[\min \{\theta(1-\tau)+\tau \omega, \theta\},(y+\sigma)(1-\tau)+\tau \omega]$. This demonstration is easy but cumbersome and the authors will gladly provide it upon request. 


\section{Proof of Proposition 3:}

The comparative static results for the finite economy are determined using (1.13):

$$
y+\sigma-k\left(\frac{N}{N-1}\right)^{M-1}=\frac{(M-1)((y+\sigma)(1-\tau)+\tau \omega)+(N-1)^{2}(\theta(1-\tau)+\tau \omega)}{(M-1)+(N-1)^{2}}
$$

We treat $M$ as a real number here (ignoring the integer constraint), as the direction for the comparative static results will be the same.

First, note that $\frac{\partial U}{\partial M}<0$, since $U^{*}(N, M)=\left(\frac{N-1}{N}\right)^{M}$ and $\frac{N-1}{N}<1$.

In general, then, for any policy parameter $x \in\{\theta, \sigma, \omega, \tau\}$, we have $\frac{\partial U}{\partial x}=-\frac{\partial M}{\partial x}$.

Now let $J=M-1$, so that $\frac{\partial M}{\partial x}=\frac{\partial(M-1)}{\partial x}=\frac{\partial J}{\partial x}$

We apply the Implicit Function Theorem to the following function, $F(J, x)$, obtained using equation (1.13) with $J=M-1$, considering a single policy parameter $x \in\{\theta, \sigma, \omega, \tau\}$ as a variable while holding all other policy parameters constant.

$$
F(J, x)=-y-\sigma+k\left(\frac{N}{N-1}\right)^{J}+\frac{J((y+\sigma)(1-\tau)+\tau \omega)+(N-1)^{2}(\theta(1-\tau)+\tau \omega)}{J+(N-1)^{2}}=0
$$

By the Implicit Function Theorem, $\frac{d J}{d x}=\frac{-\partial F / \partial x}{\partial F / \partial J}$.

Now

$$
\frac{\partial F}{\partial J}=k\left(\frac{N}{N-1}\right)^{J} \ln \left(\frac{N}{N-1}\right)+\frac{(N-1)^{2}(y+\sigma-\theta)(1-\tau)}{\left(J+(N-1)^{2}\right)^{2}}>0
$$

since $y+\sigma \geq \theta$ by assumption . 
This means that $\left|\frac{d J}{d x}\right|=-\left|\frac{\partial F}{\partial x}\right|$. So for all $x \in\{\theta, \sigma, \omega, \tau\}_{2}$ we have $\left|\frac{\partial M}{\partial x}\right|=\left|\frac{d J}{d x}\right|=-\left|\frac{\partial F}{\partial x}\right|$.

For $x=\theta, \frac{\partial F}{\partial \theta}=\frac{(N-1)^{2}(1-\tau)}{J+(N-1)^{2}}>0$ provided that $\tau<1$, so $\frac{\partial M}{\partial \theta}<0$ and $\frac{\partial U}{\partial \theta}>0$.

That is, unemployment benefits $\theta$ decrease vacancies and increase unemployment.

For $x=\sigma, \frac{\partial F}{\partial \sigma}=-1+\frac{J(1-\tau)}{J+(N-1)^{2}}=\frac{-\left(\tau J+(N-1)^{2}\right)}{J+(N-1)^{2}}<0$, so $\frac{\partial M}{\partial \sigma}>0$ and $\frac{\partial U}{\partial \sigma}<0$.

That is, employment subsidies $\sigma$ increase vacancies and decrease unemployment.

For $x=\omega, \frac{\partial F}{\partial \omega}=\frac{\tau\left(J+(N-1)^{2}\right)}{J+(N-1)^{2}}=\tau$, so $\frac{\partial M}{\partial \omega}<0$ and $\frac{\partial U}{\partial \omega}>0$ provided that $\tau>0$.

That is, the non-taxable income level $\omega$ decreases vacancies and increases unemployment.

For $x=\tau, \frac{\partial F}{\partial \tau}=\frac{-\left(J(y+\sigma-\omega)+(N-1)^{2}(\theta-\omega)\right)}{J+(N-1)^{2}}<0$ provided that $\omega \leq \theta$, which we assume. So $\frac{\partial M}{\partial \tau}>0$ and $\frac{\partial U}{\partial \tau}<0$.

That is, the tax rate $\tau$ increases vacancies and decreases unemployment.

(Note that if $\omega>\theta$ (or unemployment benefits are not taxable), the direction of the effect on vacancies and unemployment cannot be determined.) 


\section{REFERENCES}

Albrecht, J., P. Gautier, and S. Vroman, (2006) "Equilibrium Directed Search with Multiple Applications", Review of Economic Studies, 73, 869-891.

Boone, J., and L. Bovenberg, (2002) "Optimal Labour Taxation and Search", Journal of Public Economics, 85, 53-97.

Burdett, K., S. Shi, and R. Wright (2001) "Pricing and Matching with Frictions", Journal of Political Economy, 109, 1060-1085.

Hosios, A., (1990) "On the Efficiency of Matching and Related Models of Search and Unemployment”, Review of Economic Studies, 57, 279-298.

Hungerbühler, M., E. Lehmann, A. Parmentier, and B. van der Linden, (2006) "Optimal Redistributive Taxation in a Search Equilibrium Model", Review of Economic Studies, 73, 743-767.

Julien, B., J. Kennes, and I. King, (2000a) "Bidding for Labor", Review of Economic Dynamics, 3, 619-649.

Julien, B., J. Kennes, and I. King, (2000b) "Matching Foundations", University of Auckland Working Paper \# 205.

Julien, B., J. Kennes, and I. King, (2005a) "Ex Post Bidding and Efficient Coordination Unemployment”, Canadian Journal of Economics, 38, 174-193.

Julien, B., J. Kennes, and I. King, (2005b) "Implementing the Mortensen Rule", University of Otago Economics Discussion Paper 0519.

Julien, B., J. Kennes, and I. King, (2006a) "The Mortensen Rule and Efficient Coordination Unemployment", Economics Letters, 90, 149-155.

Julien, B., J. Kennes, and I. King, (2006b) "Residual Wage Disparity and Coordination Unemployment”, International Economic Review, 47, 961-989.

King, I., (2003) "A Directed Tour of Search-Theoretic Explanations for Unemployment", New Zealand Economic Papers, 37, 245-267.

Lehmann, E., and B. van der Linden, (2007) "On the Optimality of Search Matching Equilibrium When Workers Are Risk Averse", Journal of Public Economic Theory, 9, 867-884.

McAfee, P., (1993) "Mechanism Design by Competing Sellers", Econometrica, 61, 12811312. 
Mirrlees, J., (1971) "An Exploration in the Theory of Optimum Income Taxation", Review of Economic Studies, 38, 175-208.

Montgomery, J., (1991) "Equilibrium Wage Dispersion and Interindustry Wage Differentials", Quarterly Journal of Economics, 106, 163-179.

Pissarides, C., (1985) "Taxes, Subsidies, and Equilibrium Unemployment", Review of Economic Studies, 52, 121-133.

Rogerson, R., R. Shimer, and R. Wright (2005) "Search Theoretic Models of the Labor Market: A Survey", Journal of Economic Literature, XLIII, 959-9888.

Shi, S., (2008) "Search Theory: Current Perspectives", in the Palgrave Dictionary of Economics, forthcoming. 